\title{
Endovascular therapy of TASC-D lesion in critical limb ischemia
}

Ahmed Amro ${ }^{1}$, Alaa Gabi ${ }^{1}$, Mehiar El-Hamdani MD, FACC, FSCAI $^{1}$

\section{Author Affiliations:}

1. Joan C Edwards School of Medicine, Marshall University, Huntington, West Virginia

The authors have no financial disclosures to declare and no conflicts of interest to report.

\section{Corresponding Author:}

\author{
Ahmed Amro \\ Joan C Edwards School of Medicine \\ Marshall University \\ Huntington, West Virginia \\ Email: amro@marshall.edu
}




\section{Abstract}

Critical limb ischemia (CLI) is a terminal stage of peripheral artery disease (PAD); it is defined by the presence of resting pain and/or tissue loss for at least two weeks that may require urgent revascularization to promote healing and prevent limb loss. For patients with infrarenal aortoiliac occlusive disease, the revascularization options are surgery like aortofemoral bypass and axillofemoral bypass or percutaneous intervention. Aortoiliac and aortofemoral bypass procedures are associated with $74 \%$ to $95 \% 5$-year patency rates, respectively, which are comparable but not superior to percutaneous therapies. These operations may imply a significant morbidity and mortality on CLI patients who usually have multiple comorbid conditions and are considered high risk patients.

\section{Keywords}

Critical limb ischemia, TASC-D lesion, Endovascular intervention, Peripheral artery disease, Aortoiliac

\section{Case description and method}

Our patient is a 52-year-old smoker female patient with past medical history of hypertension, hyperlipidemia and severe PAD with totally occluded infrarenal abdominal aorta just distal to the renal arteries, and known right axillofemoral bypass and fem-fem bypass. She presented with bilateral leg and foot pain at rest, diminished pulses and ulceration of the legs (Rutherford class VI). ABIs were obtained and were abnormal (right leg: ABI: 0.27, TBI:0. 12. Left leg: ABI:0. 3, TBI: 0.12). CT angiography showed total occlusion of the axillo-femfem bypass, totaled infra-renal aorta with reconstitution of peripheral circulation at the level of external iliac arteries bilaterally. These findings were confirmed by angiogram obtained by accessing right radial artery, as shown in figures 1,2 and 3. 


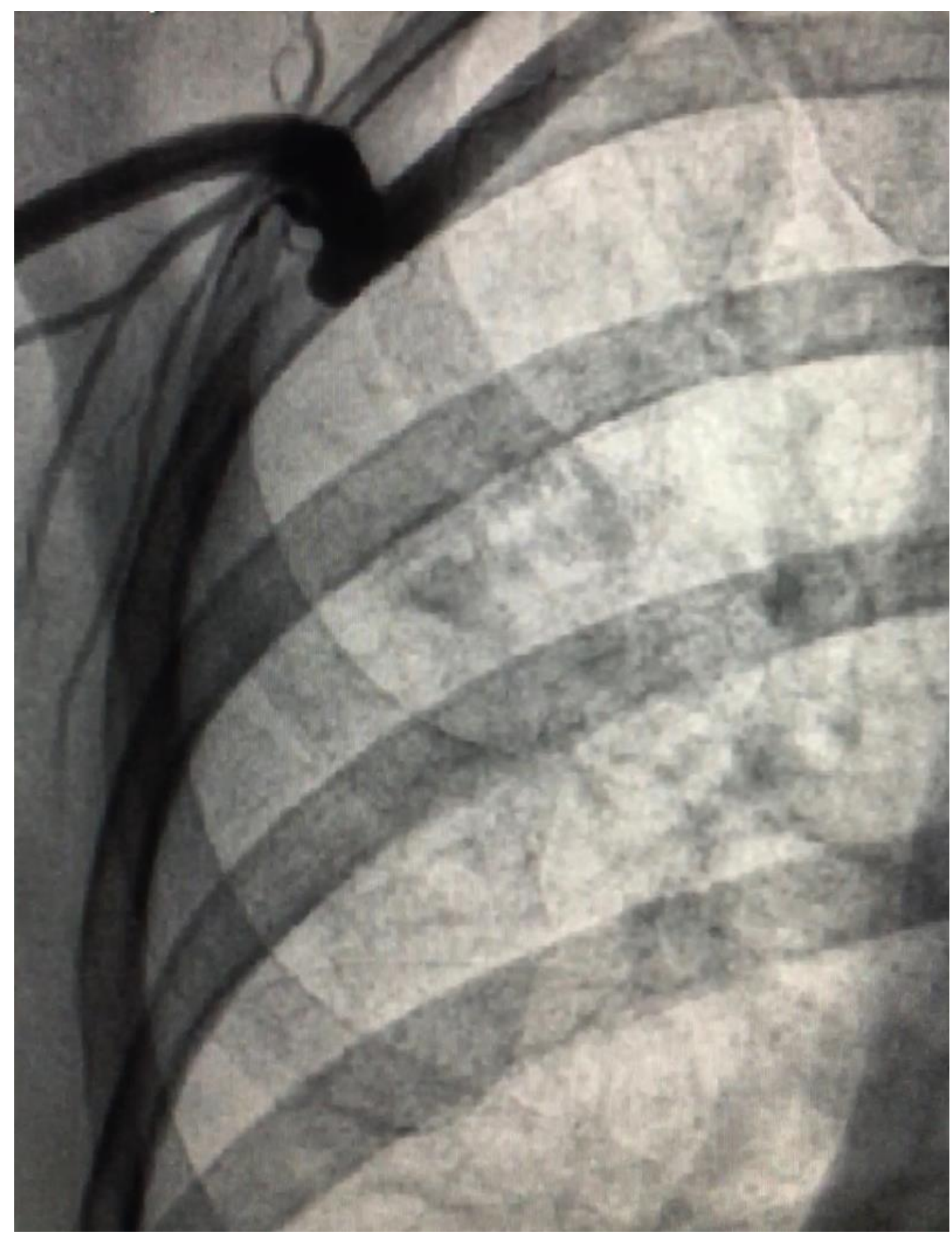

Figure 1: Angiogram of the right axillary artery showing occluded axillo-femoral bypass. 


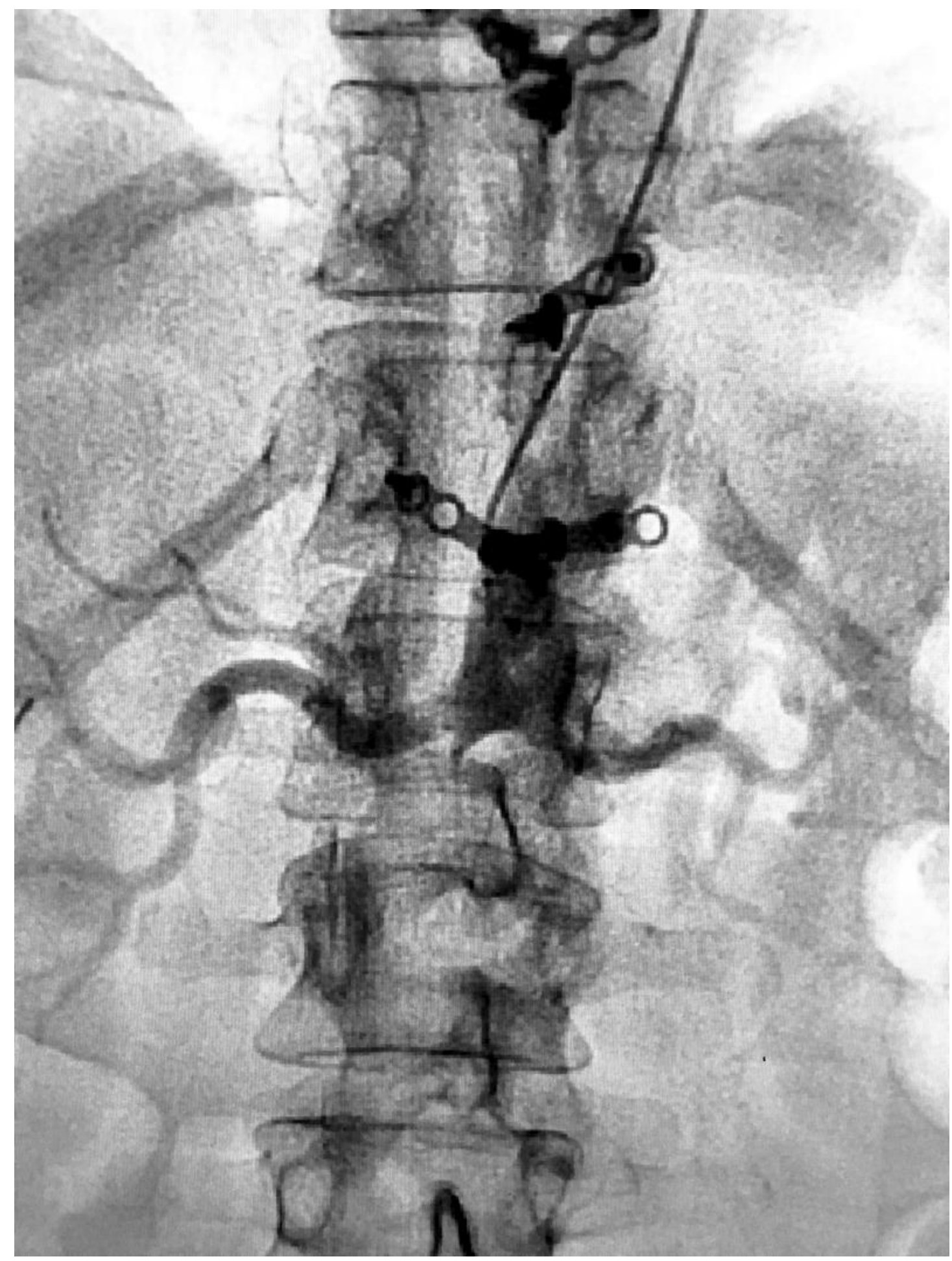

Figure 2: Aortogram showing occluded infrarenal aortic artery. 


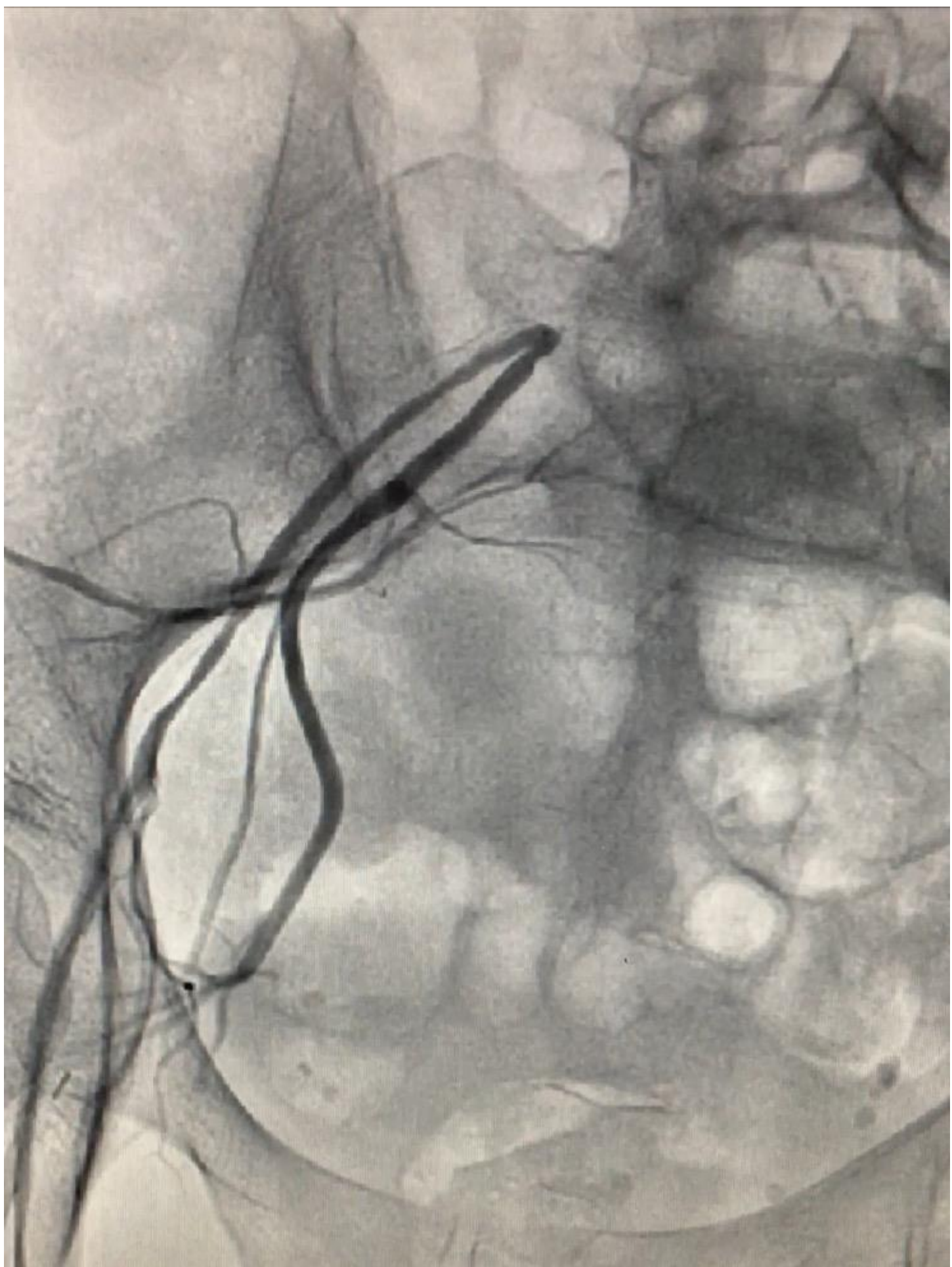

Figure 3: Angiogram right lower extremity showing reconstitution at the level right iliac artery bifurcation. Right external artery and common femoral artery are diffusely disease with multi focal stenosis. 
Using the radial access in conjunction with ultrasound guided access to the right and left femoral arteries respectively, successful PTA and stents to the abdominal aorta using self expandable stent, also to the right and left common iliac arteries using covered stents in a kissing fashion as well as treatment of the right and left external iliac arteries were successfully obtained, as shown in figures 4, 5, 6, 7, 8 and 9. After three weeks follow up, the patient's symptoms resolved, and she had good pulses bilaterally with healing ulcers and favorable ABIs (Right leg: ABI: 0.84, TBI: 0.68, Left leg: ABI: 0.82, TBI: 0.65). 


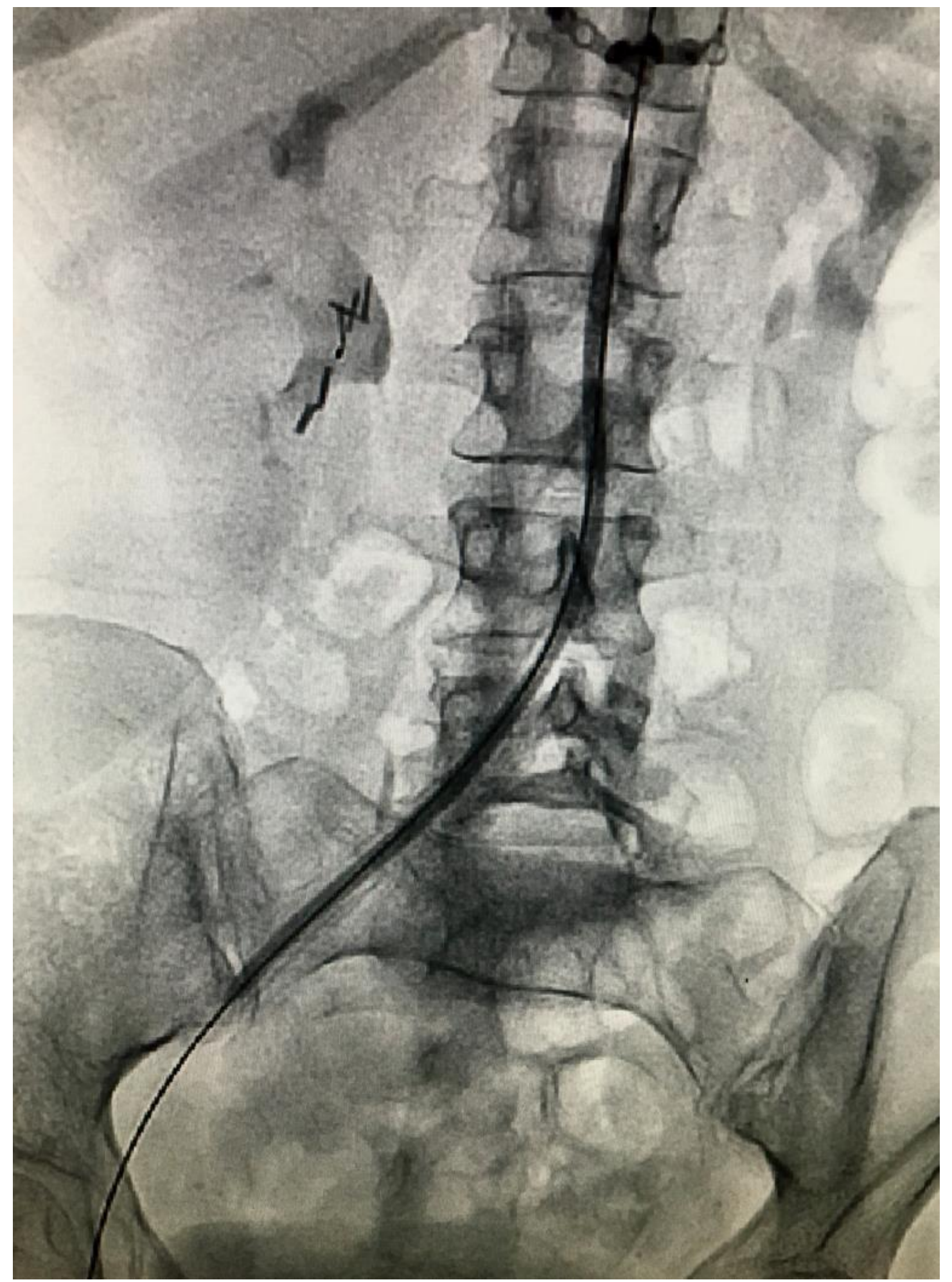

Figure 4: Balloon angioplasty of the distal abdominal aorta and right common iliac artery. 


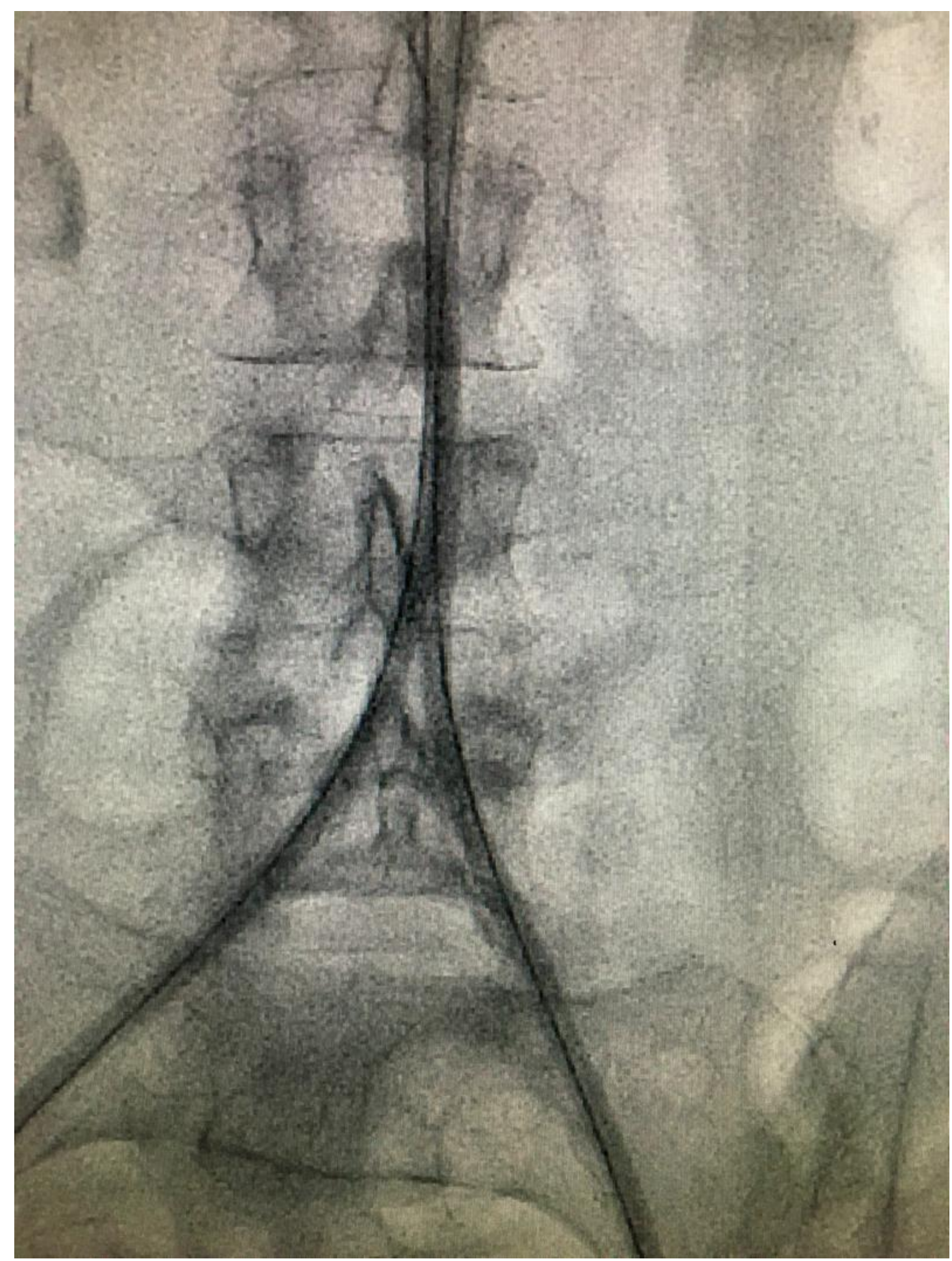

Figure 5: Balloon angioplasty of the iliac arteries extending to the aorta. 


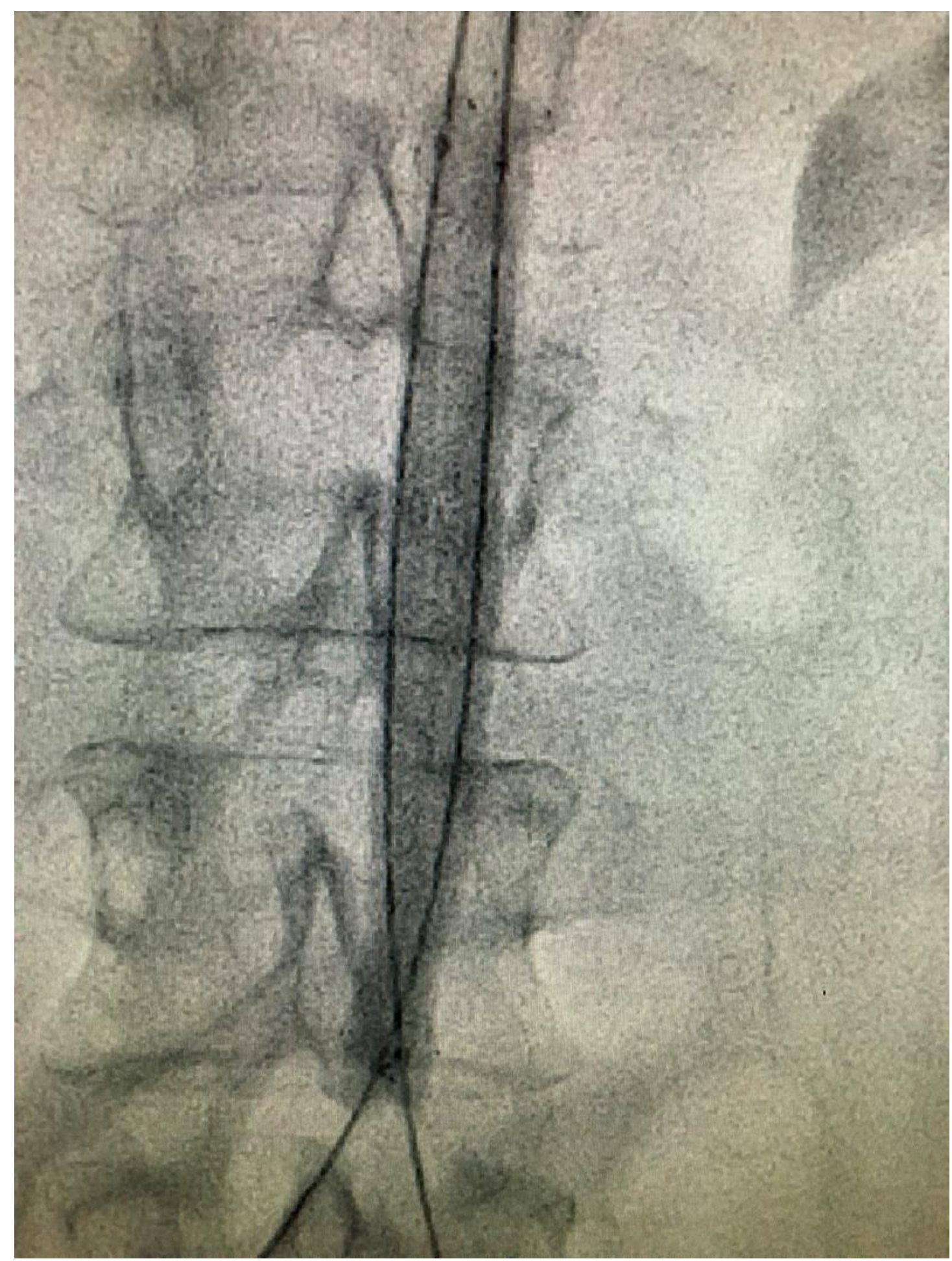

Figure 6: Stenting of the abdominal aorta using a self-expandable stent. 


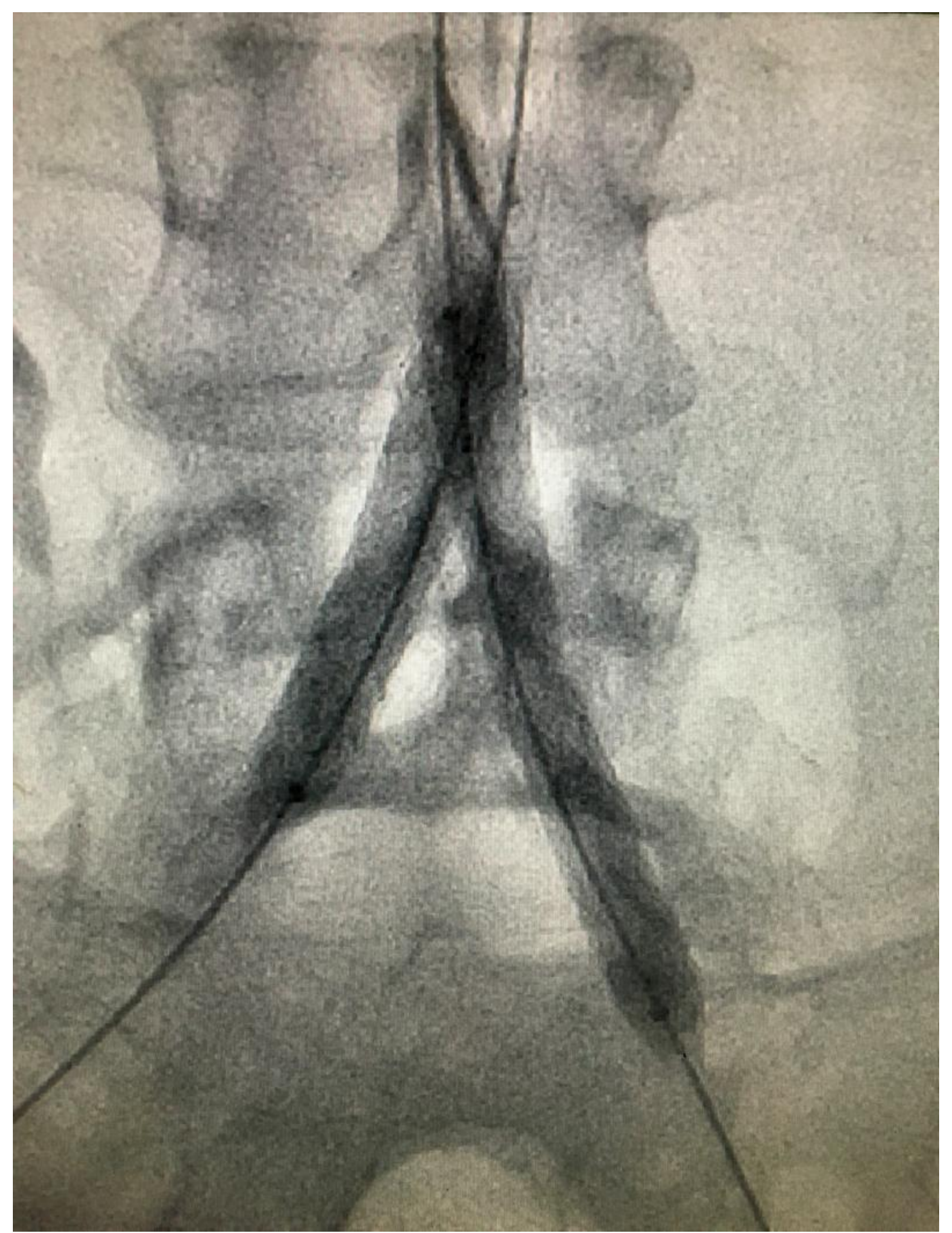

Figure 7: Stenting of the right and left iliac artery in a kissing fashion. 


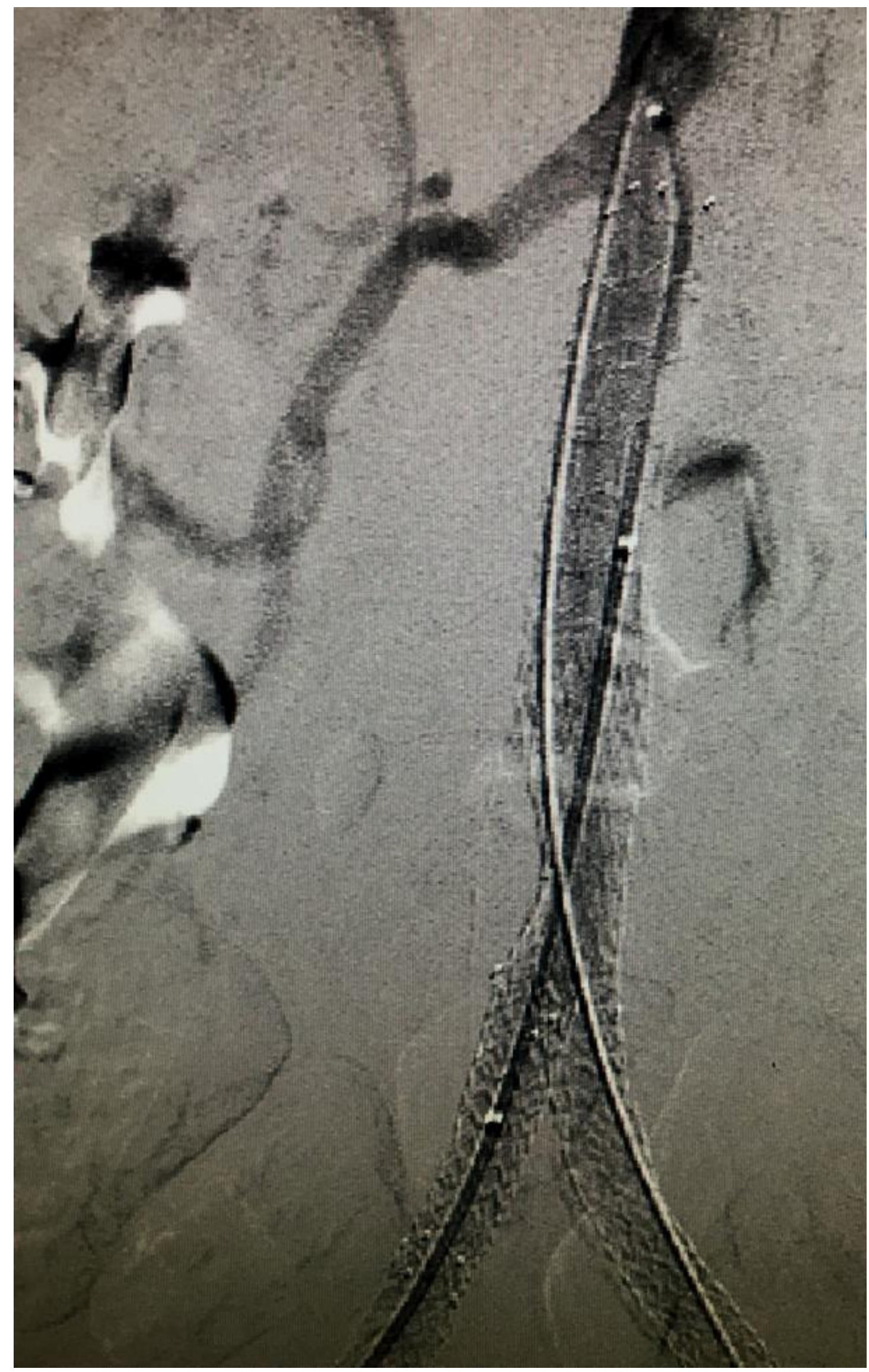

Figure 8: Angiogram image showing the abdominal aorta, right and left iliac stents after deployment. 


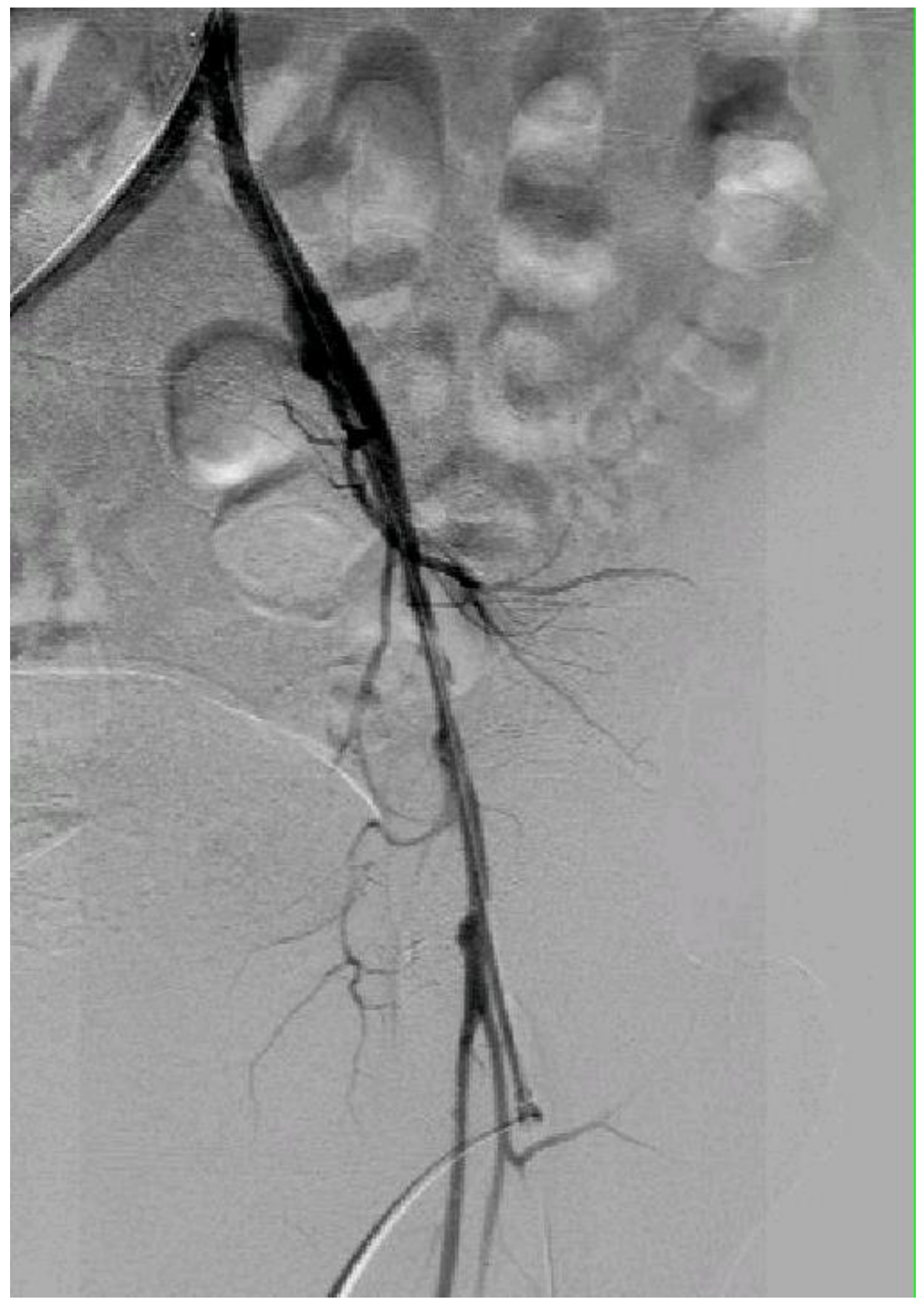

Figure 9: Angiogram image of the left lower extremity showing restoration of flow in the distal aorta bilateral common iliac, left external iliac, left common femoral to the level of left superficial femoral artery. 


\section{Discussion}

CLI (Rutherford class IV-VI) is a terminal stage of peripheral artery disease (PAD); it is defined by the presence of resting pain and/or tissue loss for at least 2 weeks. ${ }^{1}$ The target of therapy of CLI is to restore pulsatile flow to the distal limb with as low procedural morbidity as possible.

The Trans-Atlantic Inter-Society Consensus (TASC) document describes characteristic lesion morphology for ideal (type A) and unfavorable (type D) iliac lesions for endovascular therapy. ${ }^{4}$ The Bypass versus Angioplasty in Severe Ischemia of the Leg (BASIL) trial was a British multicenter randomized trial that compared an initial strategy of angioplasty with open surgery in 452 patients with CLI. Overall, this trial showed that there was no significant difference in amputation-free survival (AFS) or overall survival (OS) between the two strategies. However, for those patients who survived for at least 2 years after randomization, a bypass surgery (BSX)-first revascularization strategy was associated with a significant increase in subsequent OS and a trend towards improved AFS compared to balloon angioplasty (BAP)-first revascularization strategies. ${ }^{5}$

With advancement of technology and refinement of technical skills, endovascular therapy might be an option on a case-by-case basis. For patients with infrarenal aortoiliac occlusive disease, the revascularization options are surgery like aortofemoral bypass and axillofemoral bypass or percutaneous intervention. Aortoiliac and aortofemoral bypass procedures are associated with $74 \%$ to $95 \%$ 5-year patency rates, respectively, which are comparable but not superior to percutaneous therapies. ${ }^{2,3}$ Because many of these operations involve extensive abdominal incision, many of these operations may imply a significant morbidity and mortality on CLI patients who usually have multiple comorbid conditions and are considered high risk patients.

Endovascular treatment of aortoiliac occlusive disease has high technical success rates of 82$98 \%$, even in the setting of aortic occlusion. ${ }^{6,7}$ Due to the availability and advancement of endovascular stents, the number of aortoiliac lesions that may be treated percutaneously has been increased significantly. For common iliac bifurcation lesions, kissing balloonexpandable stents have become the preferred option. In small several series involving aortoiliac intervention, there was high success of stent placement, no major complications, symptomatic improvement, and 2-year patency rate approaching $87 \% .^{2}$ Endovascular treatment options include angioplasty and stenting; stenting has been shown to have higher patency and technical success than angioplasty, without increased complications. ${ }^{6,8}$

In our case we used a self-expanding nitinol stent, which we recommend to use in these situations because they are widely used, require a smaller sheath size (6-7 Fr.), are simply deployed, and are less likely to interfere with the renal arteries flow after deployment. Ideally, assessment of the intraluminal diameter with IVUS is recommended, as the selfexpanding nitinol stents have limited sizes up to $14 \mathrm{~mm}$. In our case, we elected to proceed directly with $14 \mathrm{~mm}$ self expandable stent based on the fact that the size of the distal aorta in women above the age of 50 is $14.6+/-1.9 \mathrm{~mm},{ }^{9}$ which seemed to be reasonable to use.

On the other hand a stent graft could be a valuable option for larger aortic diameter despite little evidence to support their use. There are several reports using stent grafts especially in active thrombosis. In atherosclerotic aortic disease, stent grafts do not increase the patency rates and theoretically increase the risk for 'toothpasting' possibly leading to embolization of 
plaque and thrombus. ${ }^{6,10}$ Plaque embolization and migration might occur proximally and distally. Furthermore, in this case the chances of renal arteries occlusion might be higher given the nature of occlusion as illustrated in the angiogram. Endovascular treatment for infrarenal aortic disease can be performed with lower morbidity than with open surgery and with better durability than extra-anatomic bypass; however, there are no randomized controlled trial data to compare.

\section{Conclusion}

Endovascular treatment of infrarenal aortoiliac occlusive disease can be performed with lower morbidity than aortofemoral bypass and with better durability than axillofemoral bypass. Patients with TASC D lesions generally will be considered surgical candidates, but with the advancement of technology and availability of several endovascular stents, endovascular therapy might be an option on a case-by-case basis. 


\section{References}

1. Slovut DP, Sullivan TM. Critical limb ischemia: medical and surgical management. Vasc Med. 2008;13(3):281-91.

2. White CJ, Gray WA. Endovascular therapies for peripheral arterial disease: an evidence-based review. Circulation. 2007;116(19):2203-15.

3. Murphy TP, Ariaratnam NS, Carney WI, Jr., Marcaccio EJ, Slaiby JM, Soares GM, et al. Aortoiliac insufficiency: long-term experience with stent placement for treatment. Radiology. 2004;231(1):243-9.

4. Norgren L, Hiatt WR, Dormandy JA, Nehler MR, Harris KA, Fowkes FG, et al. Inter-Society Consensus for the Management of Peripheral Arterial Disease (TASC II). Eur J Vasc Endovasc Surg. 2007;33 Suppl 1:S1-75.

5. Bradbury AW, Adam DJ, Bell J, Forbes JF, Fowkes FG, Gillespie I, et al. Bypass versus Angioplasty in Severe Ischaemia of the Leg (BASIL) trial: An intention-to-treat analysis of amputation-free and overall survival in patients randomized to a bypass surgery-first or a balloon angioplasty-first revascularization strategy. J Vasc Surg. 2010;51(5 Suppl):5S-17S.

6. Clair DG, Beach JM. Strategies for managing aortoiliac occlusions: access, treatment and outcomes. Expert Rev Cardiovasc Ther. 2015;13(5):551-63.

7. Kim TH, Ko YG, Kim U, Kim JS, Choi D, Hong MK, et al. Outcomes of endovascular treatment of chronic total occlusion of the infrarenal aorta. J Vasc Surg. 2011;53(6):1542-9.

8. Bosch JL, Hunink MG. Meta-analysis of the results of percutaneous transluminal angioplasty and stent placement for aortoiliac occlusive disease. Radiology. 1997;204(1):87-96.

9. Pedersen OM, Aslaksen A, Vik-Mo H. Ultrasound measurement of the luminal diameter of the abdominal aorta and iliac arteries in patients without vascular disease. J Vasc Surg. 1993;17(3):596601.

10. Klonaris C, Katsargyris A, Tsekouras N, Alexandrou A, Giannopoulos A, Bastounis E. Primary stenting for aortic lesions: from single stenoses to total aortoiliac occlusions. J Vasc Surg. 2008;47(2):310-7. 